\title{
Bi-Integrable and Tri-Integrable Couplings of a Soliton Hierarchy Associated with $\mathrm{SO}(3)$
}

\author{
Jian Zhang, ${ }^{1,2}$ Chiping Zhang, ${ }^{1}$ and Yunan Cui ${ }^{3}$ \\ ${ }^{1}$ Department of Mathematics, Harbin Institute of Technology, Harbin 150001, China \\ ${ }^{2}$ Harbin University of Science and Technology, Rongcheng Campus, Rongcheng 264300, China \\ ${ }^{3}$ Department of Mathematics, Harbin University of Science and Technology, Harbin 150080, China
}

Correspondence should be addressed to Jian Zhang; zhangjian668@hrbust.edu.cn

Received 29 November 2016; Accepted 10 May 2017; Published 4 June 2017

Academic Editor: Andrei D. Mironov

Copyright (C) 2017 Jian Zhang et al. This is an open access article distributed under the Creative Commons Attribution License, which permits unrestricted use, distribution, and reproduction in any medium, provided the original work is properly cited.

Based on the three-dimensional real special orthogonal Lie algebra $\mathrm{SO}(3)$, by zero curvature equation, we present bi-integrable and tri-integrable couplings associated with $\mathrm{SO}(3)$ for a hierarchy from the enlarged matrix spectral problems and the enlarged zero curvature equations. Moreover, Hamiltonian structures of the obtained bi-integrable and tri-integrable couplings are constructed by applying the variational identities.

\section{Introduction}

Among the well-known soliton hierarchies are the KdV hierarchy, the AKNS hierarchy, and the Kaup-Newell hierarchy [1]. The trace identity is used for constructing Hamiltonian structures of soliton equations, which is proposed by $\mathrm{Tu}$ $[2,3]$. In the case of non-semi-simple Lie algebras, integrable couplings of soliton equations are generated by zero curvature equations $[4,5]$ and the corresponding Hamiltonian structures are obtained by the variational identity [6-8].

An integrable coupling equation

$$
u_{t}=K(u)=K\left(x, t, u, u_{x}, u_{t}, u_{x x}, u_{x t}, u_{t t}, \ldots\right)
$$

is a triangular integrable system of the following form [9]:

$$
\begin{aligned}
& u_{t}=K(u), \\
& v_{t}=S(u, v),
\end{aligned}
$$

where $u$ is a function of variables $t$ and $x, u_{x}=\partial u / \partial x$, and $u_{t}=\partial u / \partial t$. If $S$ is nonlinear with respect to the second dependent variable $v$, the integrable coupling is called nonlinear.
An integrable system of the following form [10]

$$
\begin{aligned}
u_{t} & =K(u), \\
u_{1, t} & =S_{1}\left(u, u_{1}\right), \\
u_{2, t} & =S_{2}\left(u, u_{1}, u_{2}\right)
\end{aligned}
$$

is called a bi-integrable of (1).

Similarly, an integrable system of the following form [10]

$$
\begin{aligned}
u_{t} & =K(u), \\
u_{1, t} & =S_{1}\left(u, u_{1}\right), \\
u_{2, t} & =S_{2}\left(u, u_{1}, u_{2}\right), \\
u_{3, t} & =S_{3}\left(u, u_{1}, u_{2}, u_{3}\right)
\end{aligned}
$$

is called a tri-integrable of (1).

Integrable couplings correspond to non-semi-simple Lie algebras $\bar{g}$, and such Lie algebras can be written as semidirect sums [11]:

$$
\bar{g}=g \uplus g_{c}, \quad g \text {-semisimple, } g_{c} \text {-solvable. }
$$


The notion of semidirect sums $\bar{g}=g \uplus g_{c}$ means that $g$ and $g_{c}$ satisfy $\left[g, g_{c}\right] \subseteq g_{c}$, where $\left[g, g_{c}\right]=\{[A, B] \mid A \in g, B \in$ $\left.g_{c}\right\}$, with $[\cdot, \cdot]$ denoting the Lie bracket of $\bar{g}$. Obviously, $g_{c}$ is an ideal of $\bar{g}$. The subscript $c$ indicates a contribution to the construction of coupling systems. We also require the closure property between $g$ and $g_{c}$ under the matrix multiplication: $g g_{c}, g_{c} g \subseteq g_{c}$, where $g g_{c}=\left\{A B \mid A \in g, B \in g_{c}\right\}$.

Integrable couplings are useful tools for describing and explaining nonlinear phenomena of new evaluation equations. There are very rich mathematical structures behind integrable couplings. In particular, integrable couplings generalize the symmetry problem and describe other integrable properties of integrable equations. In order to enrich multicomponent integrable equations, it has been an important task to explore more integrable properties from multiintegrable couplings. For example, one can find work on the integrable couplings $[12,13]$. It is always interesting to explore any new procedure for generating integrable couplings for different soliton hierarchies, even from existing non-semisimple Lie algebras.

Recently, seeking new integrable systems including soliton hierarchies and integrable couplings forms a pretty important and interesting area of research in mathematical physics. To generate integrable couplings, bi-integrable couplings and tri-integrable couplings of soliton hierarchies, Ma proposed a new way to generate integrable couplings through a few classes of matrix Lie algebras consisting of block matrices [10]. Recently, bi-integrable couplings and triintegrable couplings for the KdV hierarchy and the AKNS hierarchy have been studied considerably [14, 15]. From $[16,17]$, bi-integrable couplings of a new soliton hierarchy associated with $\mathrm{SO}(3)$ and bi-integrable couplings of a new soliton hierarchy associated with $\mathrm{SO}(4)$ have been studied.

In this paper, we will construct bi-integrable and triintegrable couplings associated with $\mathrm{SO}(3)$ for a hierarchy from the enlarged matrix spectral problems and the enlarged zero curvature equations. Our work is essentially motivated by $[17-19]$.

\section{Bi-Integrable Couplings and Hamiltonian Structures}

2.1. Bi-Integrable Couplings Associated with $\mathrm{SO}(3)$. So as to generate bi-integrable couplings, we introduce a kind of block matrices:

$$
M_{2}\left(A, A_{1}, A_{2}\right)=\left(\begin{array}{ccc}
A & A_{1} & A_{2} \\
0 & A+\alpha A_{1} & A_{1}+\alpha A_{2} \\
0 & 0 & A+\alpha A_{1}
\end{array}\right)
$$

where $\alpha$ is an arbitrary nonzero constant and $A, A_{1}$, and $A_{2}$ are square matrices of the same order. In the following, we define the corresponding non-semi-simple Lie algebra $\bar{g}$ by a semidirect sum:

$$
\bar{g}(\lambda)=g \uplus g_{c},
$$

with

$$
\begin{aligned}
g & =\left\{M_{2}(A, 0,0) \mid A \in \widetilde{\mathrm{SO}(3)}\right\} \\
g_{c} & =\left\{M_{2}\left(0, A_{1}, A_{2}\right) \mid A_{1}, A_{2} \in \widetilde{\mathrm{SO}(3)}\right\}
\end{aligned}
$$

where the loop algebra $\widetilde{\mathrm{SO}(3)}$ is defined by

$$
\begin{aligned}
& \widetilde{\mathrm{SO}(3)}=\{A(\lambda) \in \mathrm{SO}(3) \mid \text { entries of } A(\lambda) \\
& \quad \text { - Laurent series in } \lambda\} .
\end{aligned}
$$

Obviously, we have the matrix commutator relation:

$$
\left[M_{2}\left(A, A_{1}, A_{2}\right), M_{2}\left(B, B_{1}, B_{2}\right)\right]=M_{2}\left(C, C_{1}, C_{2}\right),
$$

with $C, C_{1}$, and $C_{2}$ being defined by

$$
\begin{aligned}
C= & {[A, B], } \\
C_{1}= & {\left[A, B_{1}\right]+\left[A_{1}, B\right]+\alpha\left[A_{1}, B_{1}\right], } \\
C_{2}= & {\left[A, B_{2}\right]+\left[A_{2}, B\right]+\left[A_{1}, B_{1}\right]+\alpha\left[A_{1}, B_{2}\right] } \\
& +\alpha\left[A_{2}, B_{1}\right] .
\end{aligned}
$$

Let us consider the Lie algebra $\mathrm{SO}(3)$. It has a basis

$$
\begin{aligned}
& e_{1}=\left(\begin{array}{ccc}
0 & 0 & 1 \\
0 & 0 & 0 \\
-1 & 0 & 0
\end{array}\right), \\
& e_{2}=\left(\begin{array}{ccc}
0 & 0 & 0 \\
0 & 0 & -1 \\
0 & 1 & 0
\end{array}\right), \\
& e_{3}=\left(\begin{array}{ccc}
0 & 1 & 0 \\
-1 & 0 & 0 \\
0 & 0 & 0
\end{array}\right),
\end{aligned}
$$

with which the structure equations of $\mathrm{SO}(3)$ are $\left[e_{1}, e_{2}\right]=e_{3}$, $\left[e_{2}, e_{3}\right]=e_{1},\left[e_{3}, e_{1}\right]=e_{2}$.

The soliton hierarchy introduced in [18] has a spectral problem

$$
\phi_{x}=U \phi=U(u, \lambda) \phi, \quad u=(p, q)^{T},
$$

with the spectral matrix $U$ being chosen as

$$
U=U(u, \lambda)=\lambda e_{1}+p e_{2}+q e_{3}=\left(\begin{array}{ccc}
0 & q & \lambda \\
-q & 0 & -p \\
-\lambda & p & 0
\end{array}\right)
$$

Based on this special non-semi-simple Lie algebra $\bar{g}(\lambda)$, we begin with the corresponding enlarged spectral matrix 
$\bar{U}_{1}=M_{2}\left(U, U_{1}, U_{2}\right)$ and let supplementary spectral matrices be

$$
\begin{aligned}
& U_{1}=U_{1}\left(u_{1}, \lambda\right)=\left(\begin{array}{ccc}
0 & q^{\prime} & 0 \\
-q^{\prime} & 0 & -p^{\prime} \\
0 & p^{\prime} & 0
\end{array}\right), \quad u_{1}=\left(\begin{array}{l}
p^{\prime} \\
q^{\prime}
\end{array}\right), \\
& U_{2}=U_{2}\left(u_{2}, \lambda\right)=\left(\begin{array}{ccc}
0 & q^{\prime \prime} & 0 \\
-q^{\prime \prime} & 0 & -p^{\prime \prime} \\
0 & p^{\prime \prime} & 0
\end{array}\right), \\
& \\
&
\end{aligned}
$$

For purpose of solving the enlarged stationary zero curvature equation $\bar{V}_{1 x}=\left[\bar{U}_{1}, \bar{V}_{1}\right]$, we take $\bar{V}_{1}=M_{2}\left(V, V_{1}, V_{2}\right)$, where $V$ is defined as in [18]

$$
\begin{aligned}
V & =V(u, \lambda)=\left(\begin{array}{ccc}
0 & c & a \\
-c & 0 & -b \\
-a & b & 0
\end{array}\right) \\
& =\sum_{i \geq 0}\left(\begin{array}{ccc}
0 & c_{i} & a_{i} \\
-c_{i} & 0 & -b_{i} \\
-a_{i} & b_{i} & 0
\end{array}\right) \lambda^{-i},
\end{aligned}
$$

and the supplementary spectral matrices $V_{1}$ and $V_{2}$ read

$$
\begin{aligned}
V_{1} & =V_{1}\left(u, u_{1}, \lambda\right)=\left(\begin{array}{ccc}
0 & c^{\prime} & a^{\prime} \\
-c^{\prime} & 0 & -b^{\prime} \\
-a^{\prime} & b^{\prime} & 0
\end{array}\right) \\
& =\sum_{i \geq 0}\left(\begin{array}{ccc}
0 & c_{i}^{\prime} & a_{i}^{\prime} \\
-c_{i}^{\prime} & 0 & -b_{i}^{\prime} \\
-a_{i}^{\prime} & b_{i}^{\prime} & 0
\end{array}\right) \lambda^{-i}, \\
V_{2} & =V_{2}\left(u, u_{1}, u_{2}, \lambda\right)=\left(\begin{array}{ccc}
0 & c^{\prime \prime} & a^{\prime \prime} \\
-c^{\prime \prime} & 0 & -b^{\prime \prime} \\
-a^{\prime \prime} & b^{\prime \prime} & 0
\end{array}\right) \\
& =\sum_{i \geq 0}\left(\begin{array}{ccc}
0 & c_{i}^{\prime \prime} & a_{i}^{\prime \prime} \\
-c_{i}^{\prime \prime} & 0 & -b_{i}^{\prime \prime} \\
-a_{i}^{\prime \prime} & b_{i}^{\prime \prime} & 0
\end{array}\right) \lambda^{-i} .
\end{aligned}
$$

The enlarged stationary zero curvature equation $\bar{V}_{1 x}=$ $\left[\bar{U}_{1}, \bar{V}_{1}\right]$ is equivalent to

$$
\begin{aligned}
V_{x}= & {[U, V], } \\
V_{1 x}= & {\left[U, V_{1}\right]+\left[U_{1}, V\right]+\alpha\left[U_{1}, V_{1}\right], } \\
V_{2 x}= & {\left[U, V_{2}\right]+\left[U_{2}, V\right]+\left[U_{1}, V_{1}\right]+\alpha\left[U_{1}, V_{2}\right] } \\
& +\alpha\left[U_{2}, V_{1}\right] .
\end{aligned}
$$

The above equation system equivalently leads to

$$
\begin{aligned}
a_{x}= & p c-q b, \\
b_{x}= & -\lambda c+q a, \\
c_{x}= & \lambda b-p a, \\
a_{x}^{\prime}= & p c^{\prime}-q b^{\prime}-q^{\prime} b+p^{\prime} c+\alpha\left(p^{\prime} c^{\prime}-q^{\prime} b^{\prime}\right), \\
b_{x}^{\prime}= & -\lambda c^{\prime}+q a^{\prime}+q^{\prime} a+\alpha q^{\prime} a^{\prime}, \\
c_{x}^{\prime}= & \lambda b^{\prime}-p a^{\prime}-p^{\prime} a-\alpha p^{\prime} a^{\prime}, \\
a_{x}^{\prime \prime}= & -q b^{\prime \prime}+p c^{\prime \prime}-q^{\prime \prime} b+p^{\prime \prime} c-q^{\prime} b^{\prime}+p^{\prime} c^{\prime} \\
& +\alpha\left(-q^{\prime} b^{\prime \prime}+p^{\prime} c^{\prime \prime}\right)+\alpha\left(-q^{\prime \prime} b^{\prime}+p^{\prime \prime} c^{\prime}\right), \\
b_{x}^{\prime \prime}= & -\lambda c^{\prime \prime}+q a^{\prime \prime}+q^{\prime \prime} a+q^{\prime} a^{\prime}+\alpha q^{\prime} a^{\prime \prime}+\alpha q^{\prime \prime} a^{\prime}, \\
c_{x}^{\prime \prime}= & \lambda b^{\prime \prime}-p a^{\prime \prime}-p^{\prime \prime} a-p^{\prime} a^{\prime}-\alpha p^{\prime} a^{\prime \prime}-\alpha p^{\prime \prime} a^{\prime} .
\end{aligned}
$$

Now, we define the enlarged Lax matrices $\bar{V}_{1}^{[m]}=$ $\left(\lambda^{m} \bar{V}_{1}\right)_{+}=M_{2}\left(V^{[m]}, V_{1}^{[m]}, V_{2}^{[m]}\right), m \geq 0$, where $V^{[m]}$ is defined as $V^{[m]}=\left(\lambda^{m} V\right)_{+}$, and $V_{i}^{[m]}=\left(\lambda^{m} V_{i}\right)_{+}, i=1,2$.

Solving the enlarged zero curvature equations $\bar{U}_{1 t_{m}}-$ $\bar{V}_{1 x}^{[m]}+\left[\bar{U}_{1}, \bar{V}_{1}^{[m]}\right]=0, m \geq 0$, we get bi-integrable couplings of the soliton hierarchy in [18]

$$
\begin{aligned}
& \bar{u}_{t_{m}}=\left(\begin{array}{c}
p \\
q \\
p^{\prime} \\
q^{\prime} \\
p^{\prime \prime} \\
q^{\prime \prime}
\end{array}\right)=\left(\begin{array}{c}
-c_{m+1} \\
b_{m+1} \\
-c_{m+1}^{\prime} \\
b_{m+1}^{\prime} \\
-c_{m+1}^{\prime \prime} \\
b_{m+1}^{\prime \prime}
\end{array}\right) \\
& =\left(\begin{array}{cccccc}
0 & \frac{1}{2} & 0 & 0 & 0 & 0 \\
-\frac{1}{2} & 0 & 0 & 0 & 0 & 0 \\
0 & 0 & 0 & \frac{1}{2} & 0 & 0 \\
0 & 0 & -\frac{1}{2} & 0 & 0 & 0 \\
0 & 0 & 0 & 0 & 0 & \frac{1}{2} \\
0 & 0 & 0 & 0 & -\frac{1}{2} & 0
\end{array}\right)\left(\begin{array}{c}
-2 b_{m+1} \\
-2 c_{m+1} \\
-2 b_{m+1}^{\prime} \\
-2 c_{m+1}^{\prime} \\
-2 b_{m+1}^{\prime \prime} \\
-2 c_{m+1}^{\prime \prime}
\end{array}\right) \\
& =J_{1} P_{1, m+1} \cdot \begin{array}{llll} 
\\
0
\end{array}
\end{aligned}
$$

2.2. Hamiltonian Structures. In this section, for purpose of generating the Hamiltonian structure of hierarchy (20), we will use the corresponding variational identity [20]:

$$
\frac{\delta}{\delta \bar{u}} \int\left\{\bar{V}, \bar{U}_{\lambda}\right\} d x=\lambda^{-\gamma} \frac{\partial}{\partial \lambda} \lambda^{\gamma}\left\{\bar{V}, \bar{U}_{u}\right\}
$$


where $\{\cdot, \cdot\}$ is a required bilinear form, which is symmetric, nondegenerate, and invariant under the Lie bracket.

$\forall a=\left(a_{1}, a_{2}, a_{3}, a_{1}^{\prime}, a_{2}^{\prime}, a_{3}^{\prime}, a_{1}^{\prime \prime}, a_{2}^{\prime \prime}, a_{3}^{\prime \prime}\right) \in R^{9}, b=\left(b_{1}, b_{2}, b_{3}\right.$, $\left.b_{1}^{\prime}, b_{2}^{\prime}, b_{3}^{\prime}, b_{1}^{\prime \prime}, b_{2}^{\prime \prime}, b_{3}^{\prime \prime}\right) \in R^{9}$; we define the Lie bracket $[\cdot, \cdot]$ on $R^{9}$ as follows:

$$
\begin{aligned}
& {[a, b]=a^{T} \bar{R}_{1}(b),} \\
& \bar{R}_{1}(b) \\
& \quad=\left(\begin{array}{ccc}
R(b) & R_{1}(b) & R_{2}(b) \\
0 & R(b)+\alpha R_{1}(b) & R_{1}(b)+\alpha R_{2}(b) \\
0 & 0 & R(b)+\alpha R_{1}(b)
\end{array}\right),
\end{aligned}
$$

where

$$
\begin{aligned}
R(b) & =\left(\begin{array}{ccc}
0 & -b_{3} & b_{2} \\
b_{3} & 0 & -b_{1} \\
-b_{2} & b_{1} & 0
\end{array}\right), \\
R_{1}(b) & =\left(\begin{array}{ccc}
0 & -b_{3}^{\prime} & b_{2}^{\prime} \\
b_{3}^{\prime} & 0 & -b_{1}^{\prime} \\
-b_{2}^{\prime} & b_{1}^{\prime} & 0
\end{array}\right), \\
R_{2}(b) & =\left(\begin{array}{ccc}
0 & -b_{3}^{\prime \prime} & b_{2}^{\prime \prime} \\
b_{3}^{\prime \prime} & 0 & -b_{1}^{\prime \prime} \\
-b_{2}^{\prime \prime} & b_{1}^{\prime \prime} & 0
\end{array}\right) .
\end{aligned}
$$

Following the properties of the matrix $F_{1}: F_{1}\left(\bar{R}_{1}(b)\right)^{T}=$ $-\bar{R}_{1}(b) F_{1}$ and $F_{1}=F_{1}^{T}$, we get

$$
F_{1}=\left(\begin{array}{ccc}
\eta_{1} & \eta_{2} & 2 \eta_{3} \\
\eta_{2} & \alpha \eta_{2}+2 \eta_{3} & 2 \alpha \eta_{3} \\
2 \eta_{3} & 2 \alpha \eta_{3} & 0
\end{array}\right) \otimes\left(\begin{array}{ccc}
r_{0} & 0 & 0 \\
0 & r_{0} & 0 \\
0 & 0 & r_{0}
\end{array}\right),
$$

where $\eta_{1}, \eta_{2}, \eta_{3}, r_{0}$ are arbitrary constants. We are easy to have

$$
\operatorname{det}\left(F_{1}\right)=-64\left(\alpha^{2} \eta_{1}-\alpha \eta_{2}+2 \eta_{3}\right)^{3} \eta_{3}^{6} r_{0}^{9} \neq 0
$$

In order to get the Hamiltonian structure of the Lax integrable system, we define a bilinear form $\{a, b\}$ on $R^{9}$ of the following form:

$$
\{a, b\}=a^{T} F_{1} b .
$$

Now we can compute that

$$
\begin{aligned}
\left\{\bar{V}_{1}, \bar{U}_{1, \lambda}\right\}= & a \eta_{1}+r_{0} a^{\prime} \eta_{2}+2 r_{0} a^{\prime \prime} \eta_{3}, \\
\left\{\bar{V}_{1}, \bar{U}_{1, p}\right\}= & r_{0} b \eta_{1}+r_{0} b^{\prime} \eta_{2}+2 r_{0} b^{\prime \prime} \eta_{3}, \\
\left\{\bar{V}_{1}, \bar{U}_{1, q}\right\}= & r_{0} c \eta_{1}+r_{0} c^{\prime} \eta_{2}+2 r_{0} c^{\prime \prime} \eta_{3}, \\
\left\{\bar{V}_{1}, \bar{U}_{1, p^{\prime}}\right\}= & \left(r_{0} b+\alpha r_{0} b^{\prime}\right) \eta_{2} \\
& +\left(2 r_{0} b^{\prime}+2 \alpha r_{0} b^{\prime \prime}\right) \eta_{3}, \\
\left\{\bar{V}_{1}, \bar{U}_{1, q^{\prime}}\right\}= & \left(r_{0} c+\alpha r_{0} c^{\prime}\right) \eta_{2}+\left(2 r_{0} c^{\prime}+2 \alpha r_{0} c^{\prime \prime}\right) \eta_{3}, \\
\left\{\bar{V}_{1}, \bar{U}_{1, p^{\prime \prime}}\right\}= & \left(2 r_{0} b+2 \alpha r_{0} b^{\prime}\right) \eta_{3}, \\
\left\{\bar{V}_{1}, \bar{U}_{1, q^{\prime \prime}}\right\}= & \left(2 r_{0} c+2 \alpha r_{0} c^{\prime}\right) \eta_{3}
\end{aligned}
$$

and furthermore, we use the following formular [20]:

$$
\gamma=-\frac{\lambda}{2} \frac{d}{d \lambda} \ln |\{\bar{V}, \bar{V}\}|
$$

to obtain that $\gamma=0$. Applying the corresponding variational identity, we obtain the following Hamiltonian structure for the hierarchy of bi-integrable coupling (20):

$$
\bar{u}_{t_{m}}=\bar{K}_{1, m}(\bar{u})=\bar{J}_{1} \frac{\delta \bar{H}_{1, m}}{\delta \bar{u}} ; \quad m \geq 0,
$$

where the Hamiltonian operator is

$$
\bar{J}_{1}=\left(\begin{array}{ccc}
\eta_{1} & \eta_{2} & 2 \eta_{3} \\
\eta_{2} & \alpha \eta_{2}+2 \eta_{3} & 2 \alpha \eta_{3} \\
2 \eta_{3} & 2 \alpha \eta_{3} & 0
\end{array}\right)^{-1} \otimes\left(\begin{array}{cc}
0 & \frac{1}{2} \\
-\frac{1}{2} & 0
\end{array}\right),
$$

and the Hamiltonian functions read

$$
\bar{H}_{1, m}=-\int \frac{a_{m+2} \eta_{1}+r_{0} a_{m+2}^{\prime} \eta_{2}+2 r_{0} a_{m+2}^{\prime \prime} \eta_{3}}{m+1} d x,
$$

$m \geq 0$.

Based on (19), a direct computation yields a recursion relation:

$$
P_{1, m+1}=\bar{L}_{1} P_{1, m}
$$

where

$$
\begin{aligned}
\bar{L}_{1} & =M_{2}^{T}\left(L^{1}, L_{1}^{1}, L_{2}^{1}\right) \\
& =\left(\begin{array}{ccc}
L^{1} & 0 & 0 \\
L_{1}^{1} & L^{1}+\alpha L_{1}^{1} & 0 \\
L_{2}^{1} & L_{1}^{1}+\alpha L_{2}^{1} & L^{1}+\alpha L_{1}^{1}
\end{array}\right),
\end{aligned}
$$


with $L^{1}, L_{1}^{1}$, and $L_{2}^{1}$ being defined by

$$
\begin{aligned}
L^{1}= & \left(\begin{array}{ll}
l_{11} & l_{12} \\
l_{21} & l_{22}
\end{array}\right), \\
L_{1}^{1}= & \left(\begin{array}{ll}
l_{11}^{\prime} & l_{12}^{\prime} \\
l_{21}^{\prime} & l_{22}^{\prime}
\end{array}\right), \\
L_{2}^{1}= & \left(\begin{array}{ll}
l_{11}^{\prime \prime} & l_{12}^{\prime \prime} \\
l_{21}^{\prime \prime} & l_{22}^{\prime \prime}
\end{array}\right), \\
l_{11}= & -p \partial^{-1} q, \\
l_{12}= & \partial+p \partial^{-1} p, \\
l_{21}= & -\partial-q \partial^{-1} q, \\
l_{22}= & q \partial^{-1} p, \\
l_{11}^{\prime}= & -p \partial^{-1} q^{\prime}-\alpha p^{\prime} \partial^{-1} q^{\prime}-p^{\prime} \partial^{-1} q, \\
l_{12}^{\prime}= & p \partial^{-1} p^{\prime}+\alpha p^{\prime} \partial^{-1} p^{\prime}+p^{\prime} \partial^{-1} p, \\
l_{21}^{\prime}= & -q \partial^{-1} q^{\prime}-\alpha q^{\prime} \partial^{-1} q^{\prime}-q^{\prime} \partial^{-1} q, \\
l_{22}^{\prime}= & q \partial^{-1} p^{\prime}+\alpha q^{\prime} \partial^{-1} p^{\prime}+q^{\prime} \partial^{-1} p, \\
l_{11}^{\prime \prime}= & -p \partial^{-1} q^{\prime \prime}-\alpha p^{\prime} \partial^{-1} q^{\prime \prime}-p^{\prime} \partial^{-1} q^{\prime}-\alpha p^{\prime \prime} \partial^{-1} q^{\prime} \\
& -p^{\prime \prime} \partial^{-1} q, \\
& +q^{\prime \prime} \partial^{-1} p, \\
l_{12}^{\prime \prime}= & p \partial^{-1} p^{\prime \prime}+\alpha p^{\prime} \partial^{-1} p^{\prime \prime}+p^{\prime} \partial^{-1} p^{\prime}+\alpha p^{\prime \prime} \partial^{-1} p^{\prime} \\
& +p^{\prime \prime} \partial^{-1} p, \\
l_{21}^{\prime \prime}= & -q \partial^{-1} q^{\prime \prime}-\alpha q^{\prime} \partial^{-1} q^{\prime \prime}-q^{\prime} \partial^{-1} q^{\prime}-\alpha q^{\prime \prime} \partial^{-1} q^{\prime} \\
l_{22}^{\prime \prime}= & q \partial^{-1} p^{\prime \prime}+\alpha q^{\prime} \partial^{-1} p^{\prime \prime}+q^{\prime} \partial^{-1} p^{\prime}+\alpha q^{\prime \prime} \partial^{-1} p^{\prime} \\
&
\end{aligned}
$$

where $\partial=d / d x$ and $\partial^{-1}=\int(d / d x) d x$.

\section{Tri-Integrable Couplings and Hamiltonian Structures}

3.1. Tri-Integrable Couplings Associated with $\mathrm{SO}(3)$. So as to generate bi-integrable couplings, we introduce a kind of block matrices:

$$
\begin{aligned}
& M_{3}\left(A, A_{1}, A_{2}, A_{3}\right) \\
& =\left(\begin{array}{cccc}
A & A_{1} & A_{2} & A_{3} \\
0 & A+\beta A_{1} & \beta A_{2} & A_{1}+\beta A_{3} \\
0 & 0 & A+\beta A_{1}+\mu A_{2} & \nu A_{2} \\
0 & 0 & 0 & A+\beta A_{1}
\end{array}\right),
\end{aligned}
$$

where $\beta, \mu$, and $\nu$ are arbitrary nonzero constants and $A, A_{1}$, $A_{2}$, and $A_{3}$ are square matrices of the same order. In the following, we define the corresponding non-semi-simple Lie algebra $\bar{g}(\lambda)$ by a semidirect sum:

$$
\bar{g}(\lambda)=g \uplus g_{c},
$$

with

$$
\begin{aligned}
& g=\left\{M_{3}(A, 0,0,0) \mid A \in \widetilde{\mathrm{SO}(3)}\right\}, \\
& g_{c}=\left\{M_{3}\left(0, A_{1}, A_{2}, A_{3}\right) \mid A_{1}, A_{2}, A_{3} \in \widetilde{\mathrm{SO}(3)}\right\},
\end{aligned}
$$

where the loop algebra $\widetilde{\mathrm{SO}(3)}$ is defined by

$$
\begin{aligned}
& \widetilde{\mathrm{SO}(3)}=\{A(\lambda) \in \mathrm{SO}(3) \mid \text { entries of } A(\lambda) \\
& \text { - Laurent series in } \lambda\} .
\end{aligned}
$$

Obviously, we have the matrix commutator relation:

$$
\begin{aligned}
& {\left[M_{3}\left(A, A_{1}, A_{2}, A_{3}\right), M_{3}\left(B, B_{1}, B_{2}, B_{3}\right)\right]} \\
& \quad=M_{3}\left(C, C_{1}, C_{2}, C_{3}\right),
\end{aligned}
$$

with $C, C_{1}, C_{2}$, and $C_{3}$ being defined by

$$
\begin{aligned}
C= & {[A, B], } \\
C_{1}= & {\left[A, B_{1}\right]+\left[A_{1}, B\right]+\beta\left[A_{1}, B_{1}\right], } \\
C_{2}= & {\left[A, B_{2}\right]+\left[A_{2}, B\right]+\mu\left[A_{2}, B_{2}\right]+\beta\left[A_{1}, B_{2}\right] } \\
& +\beta\left[A_{2}, B_{1}\right], \\
C_{3}= & {\left[A, B_{3}\right]+\left[A_{3}, B\right]+\beta\left[A_{3}, B_{1}\right]+\beta\left[A_{1}, B_{3}\right] } \\
& +\left[A_{1}, B_{1}\right]+v\left[A_{2}, B_{2}\right] .
\end{aligned}
$$

We introduce the following enlarged spectral matrix to construct tri-integrable couplings for $\mathrm{SO}(3)$ hierarchy:

$$
\bar{U}_{2}=\bar{U}_{2}(\bar{u}, \lambda)=M_{3}\left(U, U_{1}, U_{2}, U_{3}\right) \in \bar{g}(\lambda),
$$

with $U=U(u, \lambda)$ being defined as in (14), where $U_{1}$ and $U_{2}$ are defined by (15), and also the supplementary spectral matrix $U_{3}$ reads

$$
\begin{aligned}
& U_{3}=U_{3}\left(u_{3}, \lambda\right)=\left(\begin{array}{ccc}
0 & q^{\prime \prime \prime} & 0 \\
-q^{\prime \prime \prime} & 0 & -p^{\prime \prime \prime} \\
0 & p^{\prime \prime \prime} & 0
\end{array}\right), \\
& u_{3}=\left(\begin{array}{l}
p^{\prime \prime \prime} \\
q^{\prime \prime \prime}
\end{array}\right) .
\end{aligned}
$$

As usual, we take a solution of the following form:

$$
\begin{aligned}
\bar{V}_{2} & =\bar{V}_{2}(\overline{\mathcal{u}}, \lambda)=M_{3}\left(V, V_{1}, V_{2}, V_{3}\right) \\
& =\left(\begin{array}{cccc}
V & V_{1} & V_{2} & V_{3} \\
0 & V+\beta V_{1} & \beta V_{2} & V_{1}+\beta V_{3} \\
0 & 0 & V+\beta V_{1}+\mu V_{2} & \nu V_{2} \\
0 & 0 & 0 & V+\beta V_{1}
\end{array}\right),
\end{aligned}
$$


where $V, V_{1}$, and $V_{2}$ are defined by (16) and (17); also $V_{3}$ reads

$$
\begin{aligned}
V_{3} & =V_{3}\left(u, u_{1}, u_{2}, u_{3}, \lambda\right)=\left(\begin{array}{ccc}
0 & c^{\prime \prime \prime} & a^{\prime \prime \prime} \\
-c^{\prime \prime \prime} & 0 & -b^{\prime \prime \prime} \\
-a^{\prime \prime \prime} & b^{\prime \prime \prime} & 0
\end{array}\right), \\
a^{\prime \prime \prime} & =\sum_{i \geq 0} a_{i}^{\prime \prime \prime} \lambda^{-i} \\
b^{\prime \prime \prime} & =\sum_{i \geq 0} b_{i}^{\prime \prime \prime} \lambda^{-i} \\
c^{\prime \prime \prime} & =\sum_{i \geq 0} c_{i}^{\prime \prime \prime} \lambda^{-i} \\
f^{\prime \prime \prime} & =\sum_{i \geq 0} f_{i}^{\prime \prime \prime} \lambda^{-i} \\
g^{\prime \prime \prime} & =\sum_{i \geq 0} g_{i}^{\prime \prime \prime} \lambda^{-i}
\end{aligned}
$$

It now follows from the enlarged stationary zero curvature equation $\bar{V}_{2 x}=\left[\bar{U}_{2}, \bar{V}_{2}\right]$ that

$$
\begin{aligned}
V_{x}= & {[U, V], } \\
V_{1 x}= & {\left[U, V_{1}\right]+\left[U_{1}, V\right]+\beta\left[U_{1}, V_{1}\right], } \\
V_{2 x}= & {\left[U, V_{2}\right]+\left[U_{2}, V\right]+\mu\left[U_{2}, V_{2}\right]+\beta\left[U_{1}, V_{2}\right] } \\
& +\beta\left[U_{2}, V_{1}\right], \\
V_{3 x}= & {\left[U, V_{3}\right]+\left[U_{3}, V\right]+\beta\left[U_{3}, V_{1}\right]+\left[U_{1}, V_{1}\right] } \\
& +\beta\left[U_{1}, V_{3}\right]+\nu\left[U_{2}, V_{2}\right] .
\end{aligned}
$$

The above equation system is equivalent to

$$
\begin{aligned}
a_{x}= & p c-q b, \\
b_{x}= & -\lambda c+q a, \\
c_{x}= & \lambda b-p a, \\
a_{x}^{\prime}= & -q b^{\prime}+p c^{\prime}-q^{\prime} b+p^{\prime} c+\beta\left(-q^{\prime} b^{\prime}+p^{\prime} c^{\prime}\right), \\
b_{x}^{\prime}= & -\lambda c^{\prime}+q a^{\prime}+q^{\prime} a+\beta q^{\prime} a^{\prime}, \\
c_{x}^{\prime}= & \lambda b^{\prime}-p a^{\prime}-p^{\prime} a-\beta p^{\prime} a^{\prime}, \\
a_{x}^{\prime \prime}= & -q b^{\prime \prime}+p c^{\prime \prime}-q^{\prime \prime} b+p^{\prime \prime} c+\mu\left(-q^{\prime \prime} b^{\prime \prime}+p^{\prime \prime} c^{\prime \prime}\right) \\
& +\beta\left(-q^{\prime} b^{\prime \prime}+p^{\prime} c^{\prime \prime}\right)+\beta\left(-q^{\prime \prime} b^{\prime}+p^{\prime \prime} c^{\prime}\right), \\
b_{x}^{\prime \prime}= & -\lambda c^{\prime \prime}+q a^{\prime \prime}+q^{\prime \prime} a+\mu q^{\prime \prime} a^{\prime \prime}+\beta q^{\prime} a^{\prime \prime}+\beta q^{\prime \prime} a^{\prime}, \\
c_{x}^{\prime \prime}= & \lambda b^{\prime \prime}-p a^{\prime \prime}-p^{\prime \prime} a-\mu p^{\prime \prime} a^{\prime \prime}-\beta p^{\prime} a^{\prime \prime}-\beta p^{\prime \prime} a^{\prime}, \\
a_{x}^{\prime \prime \prime}= & -q b^{\prime \prime \prime}+p c^{\prime \prime \prime}-q^{\prime \prime \prime} b+p^{\prime \prime \prime} c \\
& +\beta\left(-q^{\prime \prime \prime} b^{\prime}+p^{\prime \prime \prime} c^{\prime}\right)+\beta\left(-q^{\prime} b^{\prime \prime \prime}+p^{\prime} c^{\prime \prime \prime}\right) \\
& -q^{\prime} b^{\prime}+p^{\prime} c^{\prime}+v\left(-q^{\prime \prime} b^{\prime \prime}+p^{\prime \prime} c^{\prime \prime}\right), \\
b_{x}^{\prime \prime \prime}= & -\lambda c^{\prime \prime \prime}+q a^{\prime \prime \prime}+q^{\prime \prime \prime} a+\beta q^{\prime \prime \prime} a^{\prime}+\beta q^{\prime} a^{\prime \prime \prime}+q^{\prime} a^{\prime} \\
& +v q^{\prime \prime} a^{\prime \prime}, \\
c_{x}^{\prime \prime \prime}= & \lambda b^{\prime \prime \prime}-p a^{\prime \prime \prime}-p^{\prime \prime \prime} a-\beta p^{\prime \prime \prime} a^{\prime}-\beta p^{\prime} a^{\prime \prime \prime}-p^{\prime} a^{\prime} \\
& -v q^{\prime \prime} a^{\prime \prime} .
\end{aligned}
$$

Now, we define the enlarged Lax matrices $\bar{V}_{2}^{[m]}=$ $\left(\lambda^{m} \bar{V}_{2}\right)_{+}=M_{3}\left(V^{[m]}, V_{1}^{[m]}, V_{2}^{[m]}, V_{3}^{[m]}\right), m \geq 0$, where $V^{[m]}$ is defined as $V^{[m]}=\left(\lambda^{m} V\right)_{+}$, and $V_{i}^{[m]}=\left(\lambda^{m} V_{i}\right)_{+}, i=1,2,3$.

Solving the enlarged zero curvature equations $\bar{U}_{2 t_{m}}-$ $\bar{V}_{2 x}^{[m]}+\left[\bar{U}_{2}, \bar{V}_{2}^{[m]}\right]=0, m \geq 0$, we get tri-integrable couplings of the soliton hierarchy in [18]

$$
\begin{aligned}
& \bar{u}_{t_{m}}=\left(\begin{array}{c}
p \\
q \\
p^{\prime} \\
q^{\prime} \\
p^{\prime \prime} \\
q^{\prime \prime} \\
p^{\prime \prime \prime} \\
q^{\prime \prime \prime}
\end{array}\right)_{t_{m}}=\left(\begin{array}{c}
-c_{m+1} \\
b_{m+1} \\
-c_{m+1}^{\prime} \\
b_{m+1}^{\prime} \\
-c_{m+1}^{\prime \prime} \\
b_{m+1}^{\prime \prime} \\
-c_{m+1}^{\prime \prime \prime} \\
b_{m+1}^{\prime \prime \prime}
\end{array}\right) \\
& =\left(\begin{array}{cccccccc}
0 & \frac{1}{2} & 0 & 0 & 0 & 0 & 0 & 0 \\
-\frac{1}{2} & 0 & 0 & 0 & 0 & 0 & 0 & 0 \\
0 & 0 & 0 & \frac{1}{2} & 0 & 0 & 0 & 0 \\
0 & 0 & -\frac{1}{2} & 0 & 0 & 0 & 0 & 0 \\
0 & 0 & 0 & 0 & 0 & \frac{1}{2} & 0 & 0 \\
0 & 0 & 0 & 0 & -\frac{1}{2} & 0 & 0 & 0 \\
0 & 0 & 0 & 0 & 0 & 0 & 0 & \frac{1}{2} \\
0 & 0 & 0 & 0 & 0 & 0 & -\frac{1}{2} & 0
\end{array}\right)\left(\begin{array}{l}
-2 b_{m+1} \\
-2 c_{m+1} \\
-2 b_{m+1}^{\prime} \\
-2 c_{m+1}^{\prime} \\
-2 b_{m+1}^{\prime \prime} \\
-2 c_{m+1}^{\prime \prime} \\
-2 b_{m+1}^{\prime \prime \prime} \\
-2 c_{m+1}^{\prime \prime \prime}
\end{array}\right) \\
& =J_{2} P_{2, m+1} .
\end{aligned}
$$

3.2. Hamiltonian Structures. In this section, for the purpose of generating the Hamiltonian structure of the hierarchy (49), we will use the corresponding variational identity [20]:

$$
\frac{\delta}{\delta \bar{u}} \int\left\{\bar{V}, \bar{U}_{\lambda}\right\} d x=\lambda^{-\gamma} \frac{\partial}{\partial \lambda} \lambda^{\gamma}\left\{\bar{V}, \bar{U}_{u}\right\}
$$

where $\{\cdot, \cdot\}$ is a required bilinear form, which is symmetric, nondegenerate, and invariant under the Lie bracket.

$$
\forall a=\left(a_{1}, a_{2}, a_{3}, a_{1}^{\prime}, a_{2}^{\prime}, a_{3}^{\prime}, a_{1}^{\prime \prime}, a_{2}^{\prime \prime}, a_{3}^{\prime \prime}, a_{1}^{\prime \prime \prime}, a_{2}^{\prime \prime \prime}, a_{3}^{\prime \prime \prime}\right) \in R^{12},
$$
$b=\left(b_{1}, b_{2}, b_{3}, b_{1}^{\prime}, b_{2}^{\prime}, b_{3}^{\prime}, b_{1}^{\prime \prime}, b_{2}^{\prime \prime}, b_{3}^{\prime \prime}, b_{1}^{\prime \prime \prime}, b_{2}^{\prime \prime \prime}, b_{3}^{\prime \prime \prime}\right) \in R^{12}$; we define the Lie bracket $[\cdot, \cdot]$ on $R^{12}$ as follows:

$$
[a, b]=a^{T} \bar{R}_{2}(b),
$$


where

$$
\bar{R}_{2}(b)=\left(\begin{array}{cccc}
R(b) & R_{1}(b) & R_{2}(b) & R_{3}(b) \\
0 & R(b)+\beta R_{1}(b) & \beta R_{2}(b) & R_{1}(b)+\beta R_{3}(b) \\
0 & 0 & R(b)+\beta R_{1}(b)+\mu R_{2}(b) & \nu R_{2}(b) \\
0 & 0 & 0 & R(b)+\beta R_{1}(b)
\end{array}\right),
$$

with $R(b), R_{1}(b)$, and $R_{2}(b)$ being defined by (23), and

$$
R_{3}(b)=\left(\begin{array}{ccc}
0 & -b_{3}^{\prime \prime \prime} & b_{2}^{\prime \prime \prime} \\
b_{3}^{\prime \prime \prime} & 0 & -b_{1}^{\prime \prime \prime} \\
-b_{2}^{\prime \prime \prime} & b_{1}^{\prime \prime \prime} & 0
\end{array}\right) .
$$

Following the properties of the matrix $F_{2}, F_{2}\left(\bar{R}_{2}(b)\right)^{T}=$ $\bar{R}_{2}(b) F_{2}$ and $F_{2}=F_{2}^{T}$, we have

$$
\begin{aligned}
F_{2}= & \left(\begin{array}{cccc}
\eta_{1} & \eta_{2} & \eta_{3} & \eta_{4} \\
\eta_{2} & \beta \eta_{2}+\eta_{4} & \beta \eta_{3} & \beta \eta_{4} \\
\eta_{3} & \beta \eta_{3} & \mu \eta_{3}+\nu \eta_{4} & 0 \\
\eta_{4} & \beta \eta_{4} & 0 & 0
\end{array}\right) \\
& \otimes\left(\begin{array}{ccc}
r_{0} & 0 & 0 \\
0 & r_{0} & 0 \\
0 & 0 & r_{0}
\end{array}\right),
\end{aligned}
$$

where $\otimes$ is the Kronecker product and $\eta_{1}, \eta_{2}, \eta_{3}, \eta_{4}, r_{0}$ are arbitrary constants. It is easy to have

$$
\begin{aligned}
\operatorname{det}\left(F_{2}\right) & =\eta_{4}^{6}\left(\beta^{2} \eta_{1}-\beta \eta_{2}+\eta_{4}\right)^{3}\left(\mu \eta_{3}+\nu \eta_{4}\right)^{3} r_{0}^{12} \\
& \neq 0 .
\end{aligned}
$$

In order to get the Hamiltonian structure of the Lax integrable system, we define a bilinear form $\{a, b\}$ on $R^{12}$ of the following form:

$$
\{a, b\}=a^{T} F_{2} b .
$$

Now, we further compute that

$$
\begin{aligned}
\left\{\bar{V}_{2}, \bar{U}_{2, \lambda}\right\}= & -a r_{0} \eta_{1}-a^{\prime} r_{0} \eta_{2}-a^{\prime \prime} r_{0} \eta_{3}-a^{\prime \prime \prime} r_{0} \eta_{4}, \\
\left\{\bar{V}_{2}, \bar{U}_{2, p}\right\}= & r_{0} b \eta_{1}+r_{0} b^{\prime} \eta_{2}+r_{0} b^{\prime \prime} \eta_{3}+r_{0} b^{\prime \prime \prime} \eta_{4}, \\
\left\{\bar{V}_{2}, \bar{U}_{2, q}\right\}= & -r_{0} c \eta_{1}-r_{0} c^{\prime} \eta_{2}-r_{0} c^{\prime \prime} \eta_{3}-r_{0} c^{\prime \prime \prime} \eta_{4}, \\
\left\{\bar{V}_{2}, \bar{U}_{2, p^{\prime}}\right\}= & \left(r_{0} b+r_{0} b^{\prime} \beta\right) \eta_{2}+r_{0} b^{\prime \prime} \beta \eta_{3} \\
& +\left(r_{0} b^{\prime}+r_{0} b^{\prime \prime \prime} \beta\right) \eta_{4},
\end{aligned}
$$

$$
\begin{aligned}
\left\{\bar{V}_{2}, \bar{U}_{2, q^{\prime}}\right\}= & \left(-r_{0} c+\beta r_{0} c^{\prime}\right) \eta_{2}+r_{0} c^{\prime \prime} \beta \eta_{3} \\
& +\left(r_{0} c^{\prime}+\beta r_{0} c^{\prime \prime \prime}\right) \eta_{4}, \\
\left\{\bar{V}_{2}, \bar{U}_{2, p^{\prime \prime}}\right\}= & \left(r_{0} b+\beta r_{0} b^{\prime}+\mu r_{0} b^{\prime \prime}\right) \eta_{3}+\nu r_{0} b^{\prime \prime} \eta_{4}, \\
\left\{\bar{V}_{2}, \bar{U}_{2, q^{\prime \prime}}\right\}= & \left(-r_{0} c-\beta r_{0} c^{\prime}-\mu r_{0} c^{\prime \prime}\right) \eta_{3}-\nu r_{0} c^{\prime \prime} \eta_{4},
\end{aligned}
$$$$
\left\{\bar{V}_{2}, \bar{U}_{2, p^{\prime \prime \prime}}\right\}=\left(r_{0} b+\beta r_{0} b^{\prime}\right) \eta_{4} \text {, }
$$$$
\left\{\bar{V}_{2}, \bar{U}_{2, q^{\prime \prime \prime}}\right\}=\left(-r_{0} c-\beta r_{0} c^{\prime}\right) \eta_{4} \text {. }
$$

We use formula (28) and find that $\gamma=0$. Applying the corresponding variational identity, we obtain the following Hamiltonian structure for the hierarchy of tri-integrable couplings (49):

$$
\bar{u}_{t_{m}}=\bar{K}_{2, m}(\bar{u})=\bar{J}_{2} \frac{\delta \bar{H}_{2, m}}{\delta \bar{u}}, \quad m \geq 0,
$$

where the Hamiltonian operator is

$$
\begin{aligned}
\bar{J}_{2}= & \left(\begin{array}{cccc}
\eta_{1} & \eta_{2} & \eta_{3} & \eta_{4} \\
\eta_{2} & \beta \eta_{2}+\eta_{4} & \beta \eta_{3} & \beta \eta_{4} \\
\eta_{3} & \beta \eta_{3} & \mu \eta_{3}+\nu \eta_{4} & 0 \\
\eta_{4} & \beta \eta_{4} & 0 & 0
\end{array}\right)^{-1} \\
& \otimes\left(\begin{array}{cc}
0 & \frac{1}{2} \\
-\frac{1}{2} & 0
\end{array}\right),
\end{aligned}
$$

and the Hamiltonian functions read

$$
\begin{array}{r}
\bar{H}_{2, m} \\
=\int \frac{r_{0} a_{m+2} \eta_{1}+r_{0} a_{m+2}^{\prime} \eta_{2}+r_{0} a_{m+2}^{\prime \prime} \eta_{3}+r_{0} a_{m+2}^{\prime \prime \prime} \eta_{4}}{m+1} d x, \\
m \geq 0 .
\end{array}
$$

Based on (48), a direct computation shows a recursion relation:

$$
P_{2, m+1}=\bar{L}_{2} P_{2, m}
$$


where the recursion operator $\bar{L}_{2}$ is given by

$$
\begin{aligned}
\bar{L}_{2}= & M_{3}^{T}\left(L^{1}, L_{1}^{1}, L_{3}^{1}, L_{4}^{1}\right) \\
& =\left(\begin{array}{cccc}
L^{1} & 0 & 0 & 0 \\
L_{1}^{1} & L^{1}+\beta L_{1}^{1} & 0 & 0 \\
L_{3}^{1} & \beta L_{3}^{1} & L^{1}+\beta L_{1}^{1}+\mu L_{3}^{1} & 0 \\
L_{4}^{1} & L_{1}+\beta L_{4}^{1} & v L_{3}^{1} & L^{1}+\beta L_{1}^{1}
\end{array}\right),
\end{aligned}
$$

with $L^{1}$ and $L_{1}^{1}$ being given as in (34), and

$$
\begin{aligned}
& L_{3}^{1}=\left(\begin{array}{ll}
x_{11} & x_{12} \\
x_{21} & x_{22}
\end{array}\right), \\
& L_{4}^{1}=\left(\begin{array}{ll}
y_{11} & y_{12} \\
y_{21} & y_{22}
\end{array}\right), \\
& x_{11}=-\left(p+\mu p^{\prime \prime}+\beta p^{\prime}\right) \partial^{-1} q^{\prime \prime}-\beta p^{\prime \prime} \partial^{-1} q^{\prime} \\
&-p^{\prime \prime} \partial^{-1} q, \\
& x_{12}=\left(p+\mu p^{\prime \prime}+\beta p^{\prime}\right) \partial^{-1} p^{\prime \prime}+\beta p^{\prime \prime} \partial^{-1} p^{\prime}+p^{\prime \prime} \partial^{-1} p, \\
& x_{21}=-\left(q+\mu q^{\prime \prime}+\beta q^{\prime}\right) \partial^{-1} q^{\prime \prime}-\beta q^{\prime \prime} \partial^{-1} q^{\prime}-q^{\prime \prime} \partial^{-1} q, \\
& x_{22}=\left(q+\mu q^{\prime \prime}+\beta q^{\prime}\right) \partial^{-1} p^{\prime \prime}+\beta q^{\prime \prime} \partial^{-1} p^{\prime}+q^{\prime \prime} \partial^{-1} p, \\
& y_{11}=-\left(p+\beta p^{\prime}\right) \partial^{-1} q^{\prime \prime \prime}-v p^{\prime \prime} \partial^{-1} q^{\prime \prime} \\
&-\left(\beta p^{\prime \prime \prime}+p^{\prime}\right) \partial^{-1} q^{\prime}-p^{\prime \prime \prime} \partial^{-1} q, \\
&+\left(\beta q^{\prime \prime \prime}+q^{\prime}\right) \partial^{-1} p^{\prime}+q^{\prime \prime \prime} \partial^{-1} p . \\
&-\left(\beta q^{\prime \prime \prime}+q^{\prime}\right) \partial^{-1} q^{\prime}-q^{\prime \prime \prime} \partial^{-1} q, \\
& y_{22}=\left(q+\beta q^{\prime}\right) \partial^{-1} p^{\prime \prime \prime}+v q^{\prime \prime} \partial^{-1} p^{\prime \prime} \\
&+\left(\beta p^{\prime \prime \prime}+p^{\prime}\right) \partial^{-1} p^{\prime}+p^{\prime \prime \prime} \partial^{-1} p, \\
& y_{21}=\left(q+\beta q^{\prime}\right) \partial^{-1} q^{\prime \prime \prime}-v q^{\prime \prime} \partial^{-1} q^{\prime \prime} \\
& y_{12} p^{\prime \prime \prime}+v p^{\prime \prime} \partial^{-1} p^{\prime \prime}
\end{aligned}
$$

\section{Conclusion}

In this paper, we take advantage of the non-semi-simple Lie algebras consisting of $3 \times 3,4 \times 4$ block matrices and apply them to the construction of bi-integrable couplings and tri-integrable couplings associated with $\mathrm{SO}(3)$, based on the enlarged zero curvature equations. According to the associated variational identities, their Hamiltonian structures can be generated.

We can think about other related issues, for example, how we can get integrable couplings and their Hamiltonian structures when irreducible representations of $\mathrm{SO}(3)$ and $\mathrm{SO}(4)$ are used to form matrix loop algebras. In addition, we can also consider the relations between the hierarchy of triintegrable couplings associated with $\mathrm{SO}(3)$ and the hierarchy of tri-integrable couplings associated with $\mathrm{SO}(4)$.

\section{Conflicts of Interest}

The authors declare that there are no conflicts of interest regarding the publication of this paper.

\section{Acknowledgments}

This work was supported by NNSF of China (nos. 11171055 and 11471090).

\section{References}

[1] M. J. Ablowitz and P. A. Clarkson, Solitons, Nonlinear Evolution Equations and Inverse Scattering, Cambridge University Press, Cambridge, UK, 1991.

[2] G. Z. Tu, "The trace identity, a powerful tool for constructing the Hamiltonian structure of integrable systems," Journal of Mathematical Physics, vol. 30, no. 2, pp. 330-338, 1989.

[3] G. Z. Tu, "On Liouville integrability of zero-curvature equations and the Yang hierarchy," Journal of Physics. A. Mathematical and General, vol. 22, no. 13, pp. 2375-2392, 1989.

[4] W.-X. Ma, X.-X. Xu, and Y. Zhang, "Semi-direct sums of Lie algebras and continuous integrable couplings," Physics Letters. A, vol. 351, no. 3, pp. 125-130, 2006.

[5] W. X. Ma, X. X. Xu, and Y. F. Zhang, "Semidirect sums of Lie algebras and discrete integrable couplings," Journal of Mathematical Physics, vol. 47, Article ID 053501, 16 pages, 2006.

[6] Y. F. Zhang and F. K. Guo, "Two pairs of Lie algebras and the integrable couplings as well as the Hamiltonian structure of the Yang hierarchy," Chaos, Solitons Fractals, vol. 34, no. 2, pp. 490495, 2007.

[7] Y. F. Zhang, "A generalized multi-component GlachetteJohnson (GJ) hierarchy and its integrable coupling system," Chaos, Solitons Fractals, vol. 21, no. 2, pp. 305-310, 2004.

[8] Y. F. Zhang, E. G. Fan, and H. Tam, "A few expanding Lie algebras of the Lie algebra A1 and applications," Physics Letters. A, vol. 359, no. 5, pp. 471-480, 2006.

[9] W. X. Ma and B. Fuchssteiner, "Integrable theory of the perturbation equations," Chaos, Solitons and Fractals, vol. 7, no. 8, pp. 1227-1250, 1996.

[10] W. X. Ma, J. H. Meng, and H. Q. Zhang, "Integrable couplings, variational identities and Hamiltonian formulations," Global Journal of Mathematical Sciences, vol. 1, no. 1, pp. 1-17, 2012.

[11] L. Frappat, A. Sciarrino, and P. Sorba, Dictionary on Lie Algebras and Superalgebras, Academic Press, San Diego, Calif, USA, 2000.

[12] W. Ma, "Loop algebras and bi-integrable couplings," Chinese Annals of Mathematics B, vol. 33, no. 2, pp. 207-224, 2012.

[13] W.-X. Ma, "Enlarging spectral problems to construct integrable couplings of soliton equations," Physics Letters. A, vol. 316, no. 1-2, pp. 72-76, 2003.

[14] W. X. Ma, J. H. Meng, and B. Shekhtman, "Tri-integrable couplings of the KdV hierarchy associated with a non-semisimple Lie algebra," Global Journal of Mathematical Sciences, vol. 2, no. 1, pp. 9-19, 2013. 
[15] J.-H. Meng and W.-X. Ma, "Hamiltonian tri-integrable couplings of the AKNS hierarchy," Communications in Theoretical Physics, vol. 59, no. 4, pp. 385-392, 2013.

[16] S. Manukure and W.-X. Ma, "Bi-integrable couplings of a new soliton hierarchy associated with a non-semisimple Lie algebra," Applied Mathematics and Computation, vol. 245, pp. 44-52, 2014.

[17] Y. Cao, L. Chen, and B. He, "Bi-integrable couplings of a new soliton hierarchy associated with $\mathrm{SO}(4)$, , Advances in Mathematical Physics, vol. 2015, Article ID 857684, 11 pages, 2015.

[18] W.-X. Ma, "A soliton hierarchy associated with $\mathrm{SO}(3, \mathrm{R})$, , Applied Mathematics and Computation, vol. 220, pp. 117-122, 2013.

[19] B. He, L. Chen, and Y. Cao, "Bi-integrable couplings and triintegrable couplings of the modified Ablowitz-Kaup-NewellSegur hierarchy with self-consistent sources," Journal of Mathematical Physics, vol. 56, no. 1, Article ID 1.4905649, 013502, 15 pages, 2015.

[20] W.-X. Ma, "Variational identities and applications to Hamiltonian structures of soliton equations," Nonlinear Analysis: Theory, Methods \& Applications, vol. 71, no. 12, pp. e1716-e1726, 2009. 


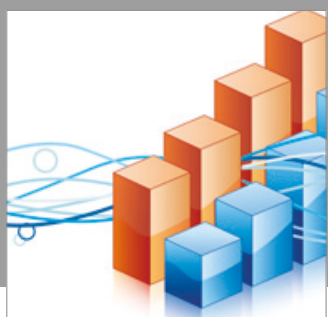

Advances in

Operations Research

vatersals

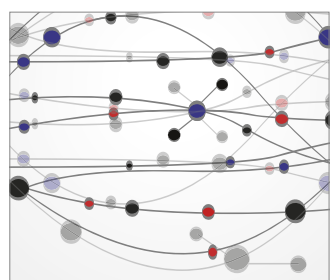

\section{The Scientific} World Journal
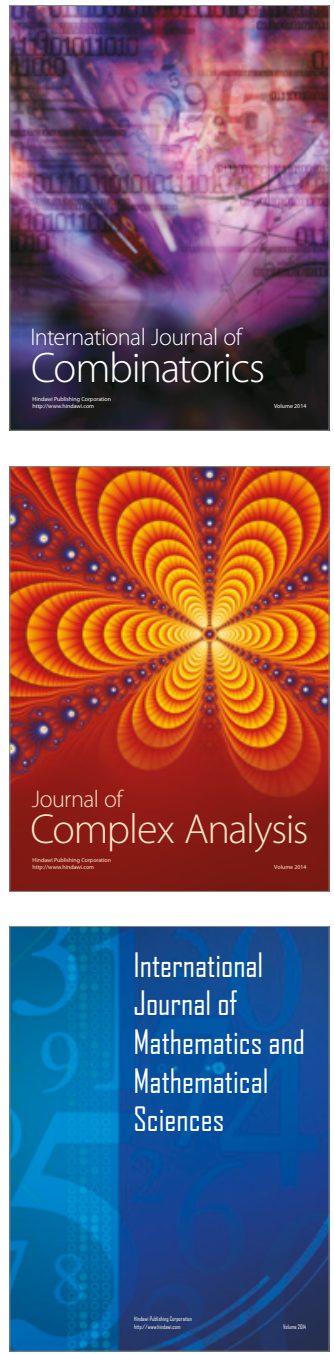
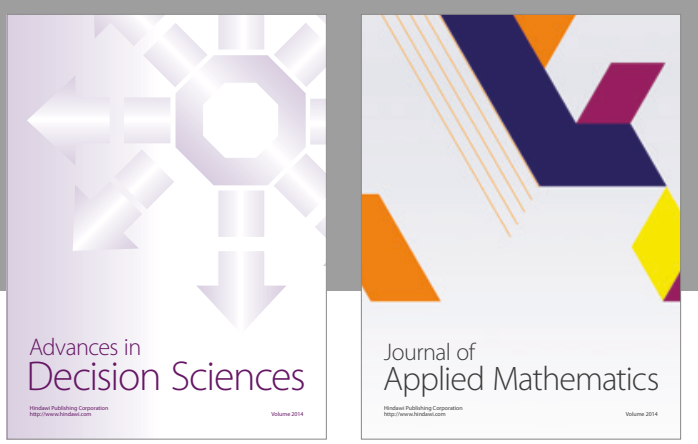

Algebra

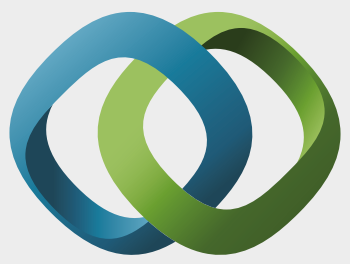

\section{Hindawi}

Submit your manuscripts at

https://www.hindawi.com
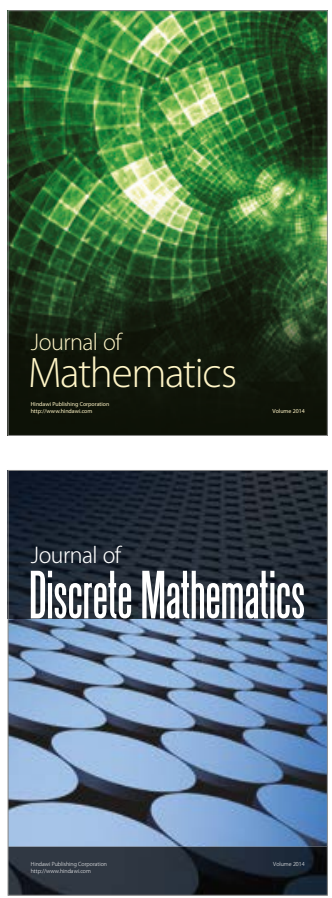

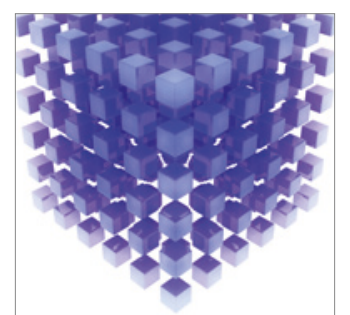

Mathematical Problems in Engineering
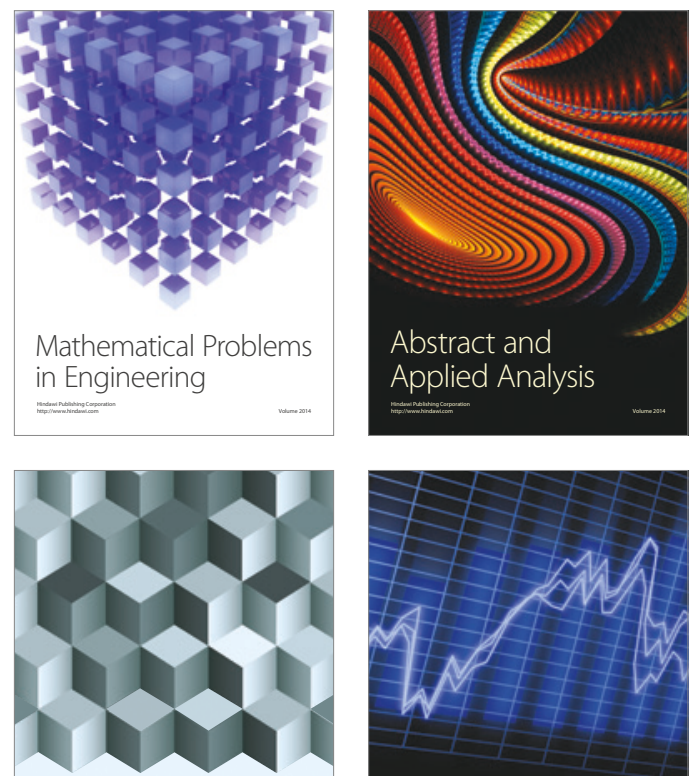

Journal of

Function Spaces

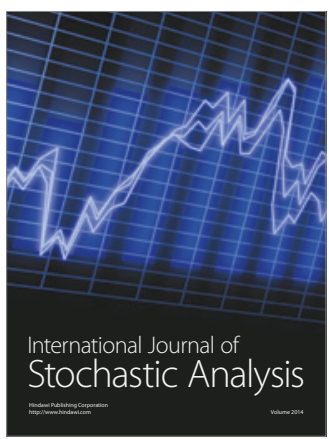

Probability and Statistics
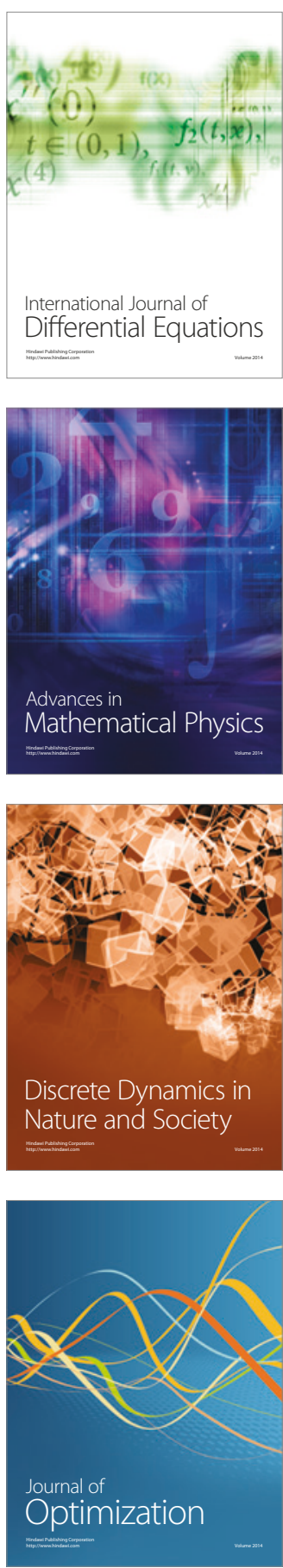